\title{
A pilot study of Auron Misheil Therapy in patients with advanced cervical cancer: Tumor response and its correlation with clinical benefit response, and preliminary quality of life data
}

\author{
JÜRGEN SCHEELE ${ }^{2,3,4}$, FAIZ NIAZI $^{2}$, JOACHIM DREVS $^{5}$, KLAUS DIERGARTEN $^{2}$ and PAPA TOURE ${ }^{1}$ \\ ${ }^{1}$ Institut de Cancerologie, CHU Le Dantec, BP5126, Dakar, Senegal; ${ }^{2}$ Auron Healthcare GmbH, \\ Merzhauser Strasse 183, D-79100 Freiburg; ${ }^{3}$ Department of Pharmacology I, Albertsrasse 25, D-79106 Freiburg; \\ ${ }^{4}$ Department of Medicine I, Hugstetterstr. 55, D-79106 Freiburg; ${ }^{5}$ Tumorklinik Sanafontis, \\ Alpine GmbH, An den Heilquellen 2, D-79111 Freiburg, Germany
}

Received March 17, 2009; Accepted May 15, 2009

DOI: $10.3892 /$ or_00000512

\begin{abstract}
This pilot study of Auron Misheil Therapy (AMT) in women with advanced cervical cancer was an open-label, single arm study to collect initial safety, efficacy, and quality of life data. Fifteen women with stage IIIb or IVa cervical cancer were given twice daily intramuscular injections of AMT (insulin, chlorpheniramine and camomile extract) for 3 months. Objective tumor response was evaluated using CT scans and analyzing the data according to the WHO RECIST criteria. Clinical Benefit Response (CBR) was assessed using a composite score comprising Karnovsky performance status, pain intensity and body weight. Safety and tolerability parameters were monitored. Quality of life was evaluated using the European Organization for Research and Treatment of Cancer Core Quality of Life Questionnaire (EORTC C-30). Eight out of 15 patients were rated as clinical responders (CBR) at 12 weeks. One patient had a partial response and 11 stable disease (WHO RECIST criteria). AMT was well tolerated. An initial analysis showed improvement in quality of life (EORTC C-30). Promising response rates, early indications of improved quality of life, and no significant safety issues mean that the second, randomized phase of the trial can be initiated with a longer treatment duration. Patients with advanced cervical cancer showed positive clinical responses to Auron Misheil Therapy. The treatment was well tolerated, with indications of improved quality of life.
\end{abstract}

\section{Introduction}

Auron Misheil Therapy contains human insulin and chlorpheniramine at low therapeutic doses, and aqueous camomile

Correspondence to: Dr J. Scheele, Auron Healthcare GmbH, Merzhauser Strasse 183, D-79100 Freiburg, Germany

E-mail: j.scheele@auronhealthcare.com

Key words: auron mishiel therapy, cervical cancer, quality of life extract. It has been developed for intramuscular use in patients with malignancies and viral infections. It has been given to end-stage cancer patients, on a compassionate use basis, since 1989. From the documentation available from these patients, there was no indication of tumor reduction, but many patients reported an improved quality of life and were more able to perform daily activities, occasionally accompanied by weight gain and pain reduction.

Preclinical toxicological investigations did not raise any safety concerns (Caballero et al, unpublished data). Animal models have shown borderline antitumor activity (1). The cervical cancer cell line DOTC2 was the most sensitive model in a monolayer proliferation assay. In experiments to assess the immuno-modulatory effect of AMT, there was a dosedependent release of IL- 6 and TNF- $\alpha$ from peripheral blood mononuclear cells taken from healthy volunteers, probably indicating activation of monocytes and B-lymphocytes. In another experiment, AMT selectively induced marker expression in resting natural killer cells, monocytes and B-cells, but not T-cells.

However, the proliferation of pre-activated B- and T-cells was inhibited. This pharmacological pattern indicates that AMT might enhance anti-tumor and anti-viral immune responses. AMT caused a super-induction of cytokine production (IL-6, IL-8 and TNF- $\alpha$ ) in monocytes that had been prestimulated by lipopolysaccharide (LPS). The effect was higher with LPS and AMT together than with either reagent alone. LPS was used at the maximum stimulatory concentration, which suggests that AMT was triggering a different stimulatory pathway. This could be a useful effect to enhance a weak immune response at sites of local inflammation such as a tumor or an infection. Preclinical toxicological investigations did not raise any safety concerns.

Research on the components of AMT has shown that they each may contribute to its activity in patients with cancer. The active constituents of aqueous camomile extract are flavonoids, polysaccharides and phenolic compounds (2). The main flavonoids in aqueous camomile extract are apigenin, quercetin and luteolin. Flavonoids are known to have anti- 
allergic, hepatoprotective, antithrombotic, anti-inflammatory, antiviral and anticarcinogenic properties, act as free radical scavengers and are powerful antioxidants. Aqueous camomile extracts contain phenolic compounds, such as ferulic and chlorogenic acid. Antiproliferative effects of these substances have been described $(3,4)$, as well as radical scavenging activity (5). A chemopreventive effect on colorectal carcinogenesis has been shown for both ferulic and chlorogenic acid (6).

In cancer therapy, chlorpheniramine, an antihistamine, can be used to treat hypersensitivity to cytostatic and cytotoxic drugs (7). Antihistamines can also be used as adjuncts in the treatment of cancer pain, as they have analgesic effects (8). An antiproliferative effect has been shown in vitro in human breast cancer cell lines, probably mediated by an inhibition of ornithine decarboxylase synthesis (9). In mice the growth of Ehrlich carcinoma is inhibited (10). A chemopreventive effect of chlorpheniramine in colon cancer has been discussed after showing an inhibition of colon aberrant crypt formation in rats (11). Chlorpheniramine blocks HERG channels (12), which may impair growth of HERG-bearing tumor cells (13) and may explain the antiproliferative effects of chlorpheniramine seen in some tumor models.

Cancer patients, due to the high energy requirements of tumors, often have changes in their carbohydrate metabolism, characterized by a higher glucose production, glucose intolerance, increased whole-body glucose turnover rate and insulin resistance $(14,15)$. Glucose intolerance is one of the earliest recognized metabolic alterations in patients with cancer (15). Insulin treatment may have two beneficial mechanisms of action. Firstly, it may potentiate the effects of chemotherapeutic agents by increasing the fluidity and permeability of cellular membranes and thus increasing the uptake of these agents into cells (16), and secondly, it may partially reverse tumor-induced cachexia. A randomized clinical trial in patients with multidrug-resistant, metastatic breast cancer showed that combined treatment with insulin and methotrexate resulted in a significantly higher number of patients with stable disease and overall a significantly lower increase in tumor size than with each drug alone $(17,18)$.

Cachexia, characterized by marked weight loss and wasting of skeletal muscle mass and adipose tissue, is an important factor leading to early death of cancer patients (19). In cachectic tumor-bearing rats, lipid and cholesterol metabolic alterations were partially reversed by insulin, resulting in increased body weight, food intake and a prolonged survival time, without stimulating tumor growth (20). Insulin limited the use of endogenous substrates, preserved food intake, and promoted the use of exogenous substrates for energy expenditure. In patients with lymphoma, insulin seemed to counteract the imbalance between glucose uptake of tumor and muscle, circumventing tumor-induced metabolic abnormalities (21). In cachectic cancer patients, insulin together with hypercaloric parenteral nutrition improved skeletal muscle protein synthesis and whole-body protein net balance (18).

A Phase I placebo-controlled, single-dose study carried out in healthy volunteers did not raise any safety concerns, and the pharmacokinetic analysis of insulin and chlorpheniramine produced the expected results (24).
The aim of this pilot study was to examine the efficacy, safety, and effect on quality of life of AMT in patients with advanced cervical cancer who have a poor prognosis. In these patients, quality of life is a critical issue. This is the first phase of a 2-stage study to ascertain the feasibility of performing a larger-scale, randomized study in the same patient population.

\section{Patients and methods}

This was an open-label, single arm pilot study to collect initial safety, efficacy, and quality of life data before a larger, randomized trial is conducted. The study was carried out at the cancer institute in Dakar, Senegal from November 2005 to April 2006; approval was obtained from the appropriate authority and the responsible ethics committee in Senegal.

Study population. Fifteen adult women (18 years or older) with histologically confirmed advanced cervical cancer were recruited to this pilot, Phase II clinical study. The cancer had to be stage IIIb or IVa; stage II was only allowed if the patient had relapsed. Patients were to be recruited only if the cancer was unlikely to respond to existing therapy, was refractory to existing therapy or if no other therapy was available at the study site. Patients had to have measurable disease by CT scan, be HIV-negative, have a life expectancy of $>3$ months and have given written informed consent. Patients were put on stable analgesia for 7 days before inclusion in the study; once in the study any changes in analgesic medication were recorded.

Study assessments. An important objective was to assess the tumor response according to WHO-RECIST criteria. Objective tumor response was evaluated using CT scans at screening and at 12 weeks and analyzing the data according to WHORECIST criteria (22). Measurable lesions were those that could be accurately measured in at least 1 dimension and were $\geq 20 \mathrm{~mm}$ with conventional techniques or $\geq 10 \mathrm{~mm}$ with a spiral CT scan. If there was only 1 measurable lesion, the malignancy had to be confirmed by cytology or histology. Up to 5 measurable lesions were identified as target lesions and their longest diameters were recorded. The sum of the longest diameter of the target lesions was the reference to calculate the objective tumor response as follows: complete response (CR), disappearance of all target lesions; partial response (PR), a $\geq 30 \%$ decrease in the sum of the longest dimensions of the target lesions compared to baseline; progressive disease (PD), a $\geq 20 \%$ increase in the sum of the longest dimensions of the target lesions compared to baseline, or the appearance of 1 or more new lesions; stable disease (SD), neither CR, PR or PD.

Local reactions (pain, redness, swelling) were documented in patient diaries. Laboratory tests, ECG and vital signs were also monitored. The tumor marker, squamous cell carcinoma antigen (SCC) was measured at baseline and at 12 weeks. Although an objective of the study was to assess the Clinical Benefit Response (CBR) at 4,8 and 12 weeks, a clinical measure for the symptom improvement, we will focus on data for the correlation of tumor response with CBR in this study. 
Table I. Demographic data and tumor staging.

\begin{tabular}{lcc}
\hline Demographic data $(\mathrm{n}=15)$ & Mean \pm SD & Range \\
\hline Age (years) & $51 \pm 8$ & $38-63$ \\
Height $(\mathrm{cm})$ & $163 \pm 4$ & $157-173$ \\
Weight $(\mathrm{kg})$ & $57.5 \pm 9.5$ & $45-82$ \\
No. of pregnancies & 9.4 & $3-13$ \\
No. of deliveries & 8.6 & $1-13$ \\
& & \\
Tumor staging & No. of patients & \\
(FIGO classification) $(\mathrm{n}=15)$ & & \\
IIb & 1 & \\
IIIa & 2 & \\
IIIb & 4 & \\
IVa & 7 & \\
IVb & 1 (after scan) \\
\hline
\end{tabular}

The CBR is a composite score of the performance status (Karnofsky performance status), pain intensity and body weight. The Karnofsky performance status was assessed at screening, weekly during month 1 and then monthly. This parameter was graded as positive if there was $a \geq 10 \%$ improvement from screening, negative if there was a $>10 \%$ worsening, and otherwise as stable.

Pain intensity (which excluded local pain at the injection site) was assessed at screening and daily during treatment using a $10-\mathrm{cm}$ visual analog scale, with $0 \mathrm{~cm}$ representing no pain and $10 \mathrm{~cm}$ the worst possible pain. This parameter was graded as positive if there was a $>25 \%$ improvement (the baseline value had to be $\geq 2 \mathrm{~cm}$ ), negative if there was any worsening, and otherwise as stable. Body weight was assessed at screening and daily during treatment. This parameter was graded as positive if there was a weight gain of $\geq 5 \%$, negative if there was weight loss of $\geq 5 \%$, and otherwise stable. For pain and body weight, the mean of the weekly results was used; if $>3$ values were missing, the mean value was considered missing.

The CBR was then defined as follows: clinical responder, at least one parameter was positive and the others stable; clinical non-responder, at least one parameter was negative; clinically stable, all 3 parameters stable.

Quality of life was evaluated using the European Organization for Research and Treatment of Cancer Core Quality of Life Questionnaire (EORTC C-30) at screening, weekly during the first month and at 8 and 12 weeks (23). This is a validated and well established scoring instrument in which the questions are grouped to form subscores for functional status (physical, role, cognitive, emotional, and social), symptomatology (fatigue, pain, nausea and vomiting), and global health status. In this preliminary analysis, the scores for questions 1 to 28 are summarized separately from the two questions on global health and quality of life, 29 and 30. In the first summary, a higher score represents a lower quality of life. For the last two questions, a higher score represents a higher quality of life.
Auron GmbH (MCS Microcarrier Systems GmbH, Neuss, Germany). For study 1 (batch-no.) AMT 2003 Cam (5228), AMT 2003 Vit(+) (5229) and AMT 2003 Ins (5230) was used. For study 2 AMT 2003 Cam (6091), AMT 2003 Vit(+) (6090), and AMT zero IL6 (AMT0, 6089) was used. All components were stored at $4^{\circ} \mathrm{C}$.

Preparation of compound. AMT was prepared by mixing defined amounts of the three individually supplied components. For $10 \mathrm{ml}$ of AMT $5 \mathrm{ml}$ AMT $2003 \mathrm{Cam} \mathrm{H}$ were transferred into an empty mixing vial. AMT 2003 Vit(+) $(4.75 \mathrm{ml})$ and $0.25 \mathrm{ml}$ AMT $2003 \mathrm{Ins}$ were added. All three components were mixed thoroughly. This procedure was performed prior to each application.

An AMT-application contains insulin $(2.5 \mathrm{IU} / \mathrm{ml})$ and chlorpheniramine $(0.813 \mathrm{mg} / \mathrm{ml})$ together with aqueous chamomile extract. Patients were treated for up to 3 months with $0.066 \mathrm{ml} / \mathrm{kg}$ (up to a maximum volume of $5 \mathrm{ml}$ ) AMT given as an intramuscular injection twice daily. The injections were given at the study center (the first 2 injections were given while the patients were hospitalized at the study site), and very occasionally at a patient's home, by qualified and trained staff.

Statistical analysis. As this was a pilot study, only descriptive statistics were used in the analysis. No hypothesis testing was done.

Immune monitoring. It was well known from preclinical assessments that AMT has immuno-modulatory activities. An important aspect of the present trial was thus to assess whether these properties were also to be seen in patients. Blood samples were taken at the screening, visit 1 (5-10 days after start of treatment) and visit 2 (21-42 days after start of treatment). For logistical reasons (cells have to be analysed freshly and cannot be frozen), a time window and not a fixed date was given for the visits to ship as many samples at a time as possible. The following four analyses were to be performed with the samples. Serum, interleukin-6 (IL-6); isolated blood cells: i) stimulation of monocytes and $\mathrm{T}$ cells followed by quantitation of the following cytokines: IL-2, IL-4, IL-6, IL-10, TNF- $\alpha$, IFN- $\gamma$ for T cells (after stimulation with CD3 and CD28) and IL-1ß, IL-6, IL-8, IL-10, IL-12p70, TNF- $\alpha$ for monocytes (after stimulation with LPS); ii) stimulation of T-cells with $\mathrm{CD} 3 / \mathrm{CD} 28$ and measurement of $\mathrm{T}$ cell proliferation after $72 \mathrm{~h}$.

\section{Results}

Fifteen women were recruited to and completed the study. Demographic data are summarized in Table I. All patients received the full 3 months of treatment. All patients had a primary tumor; there were no relapsers. Only one patient had received prior chemotherapy; for the other 14 patients the tumor was either unlikely to respond to chemotherapy or no therapy was available at the study site. Tumor staging is shown in Table I. Four patients were enrolled who did not satisfy the inclusion criterion for tumor staging. Three patients had less advanced cervical cancer (IIb and IIIa) and one was originally classified as stage IVa but this changed to IVb after a scan. 
Table II. Tumor response according to WHO-RECIST criteria.

\begin{tabular}{lcccc}
\hline $\begin{array}{l}\text { Patients } \\
\text { no. }\end{array}$ & $\begin{array}{c}\text { Sum of longest diameter of all target } \\
\text { lesions at screening }(\mathrm{mm})\end{array}$ & $\begin{array}{c}\text { Sum of longest diameter of all target } \\
\text { lesions after } 3 \text { months }(\mathrm{mm})\end{array}$ & Difference in \% & Outcome \\
\hline 101 & 104 & 107 & 2.9 & Progressive \\
102 & 65 & 63 & -3.1 & Stable \\
103 & 97 & 92 & -5.2 & Stable \\
104 & 119 & 119 & 0 & Stable \\
105 & 106 & 95 & -10.4 & Stable \\
106 & 185 & 151 & -18.4 & Stable \\
107 & 60 & 67 & 11.7 & Stable \\
108 & 113 & 86 & -23.9 & Stable \\
109 & 180 & 210 & 16.7 & Stable \\
110 & 97 & 100 & 3.1 & Progressive \\
111 & 92 & 109 & 18.5 & Stable \\
112 & 40 & 43 & 7.5 & Stable \\
113 & 83.4 & 50.3 & -39.7 & Partial response \\
114 & 64 & 83 & 29.7 & Progressive \\
115 & 70 & 63 & -10 & Stable \\
\hline
\end{tabular}

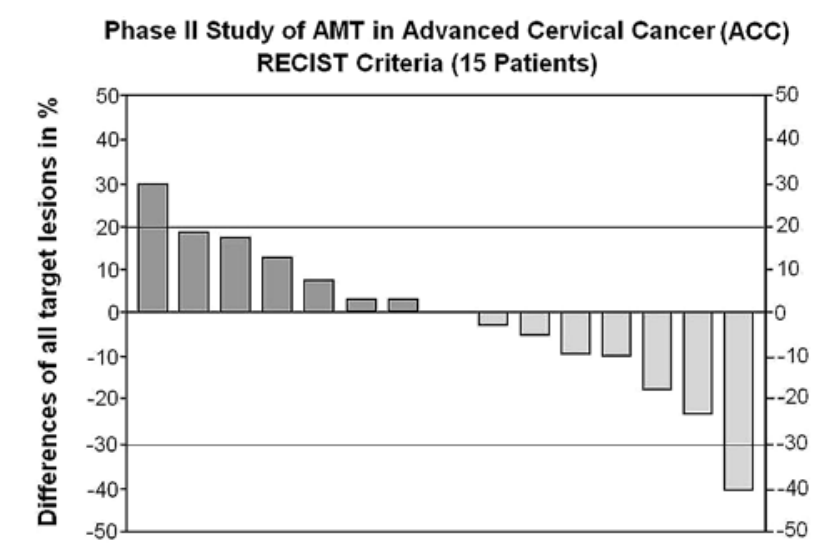

Figure 1. Waterfall plot for target lesions of the 15 patients according to RECIST criteria.

The patients which were staged as IIb and IIIa qualified under the criterion that "no therapy was available at the study site'. The assessment of clinical status of the patients at screening showed that the Karnofsky performance status was high at screening, and that there was a range of pain intensity from none ( 2 patients scored 0 ) to severe pain ( 3 patients scored 9).

Safety results. Seven patients experienced 18 adverse events. The most common adverse events were gastrointestinal, with vomiting reported 6 times in 5 patients and diarrhea by 3 patients. Other events reported were urinary tract infection (in 2 patients), malaria, genital infection, high blood pressure, creatine kinase elevation, fever and anorexia. All events except one were considered to be unrelated or unlikely related to the study drug. One patient had moderate hypoglycemia that was considered to be probably related to study drug. The patient recovered the same day. Most of the adverse events
Table III. SCC tumor marker values.

\begin{tabular}{lrrl}
\hline $\begin{array}{l}\text { Patients } \\
\text { no. }\end{array}$ & $\begin{array}{c}\text { SCC at } \\
\text { screening }(\mu \mathrm{g} / \mathrm{l})\end{array}$ & $\begin{array}{c}\text { SCC after } \\
\text { months }(\mu \mathrm{g} / \mathrm{l})\end{array}$ & $\begin{array}{l}\text { Tumor response } \\
\text { according to } \\
\text { WHO-RECIST }\end{array}$ \\
\hline 101 & 20.7 & 62.1 & Progressive \\
102 & 11.8 & 20.5 & Stable \\
103 & 29.7 & N/A & Stable \\
104 & 59.8 & 59.9 & Stable \\
105 & 228 & 252 & Stable \\
106 & 3.4 & 4.5 & Stable \\
107 & 8.0 & 7.8 & Stable \\
108 & 14.1 & 22.1 & Stable \\
109 & 0.7 & 0.5 & Stable \\
110 & 11.8 & 13.5 & Progressive \\
111 & 7.0 & 15.3 & Stable \\
112 & $<0.5$ & $<0.5$ & Stable \\
113 & 2.9 & 7.6 & Partial response \\
114 & 8 & 17.8 & Progressive \\
115 & 10.4 & 12.3 & Stable \\
\hline
\end{tabular}

N/A, Not available.

were moderate in intensity and none of the events resulted in a change in the dose or discontinuation of study drug.

Local reactions were reported in 14 of the 15 patients enrolled. Most patients had occasional local pain (reported on 1 to 2 days) during the 3 months of treatment, and 2 patients had local swelling. No reddening was reported. Only one patient had more consistent reporting of local pain (patient 107) with pain reported on 31 days. There were no clinically significant findings in the laboratory tests, ECG and vital signs (data not shown). 


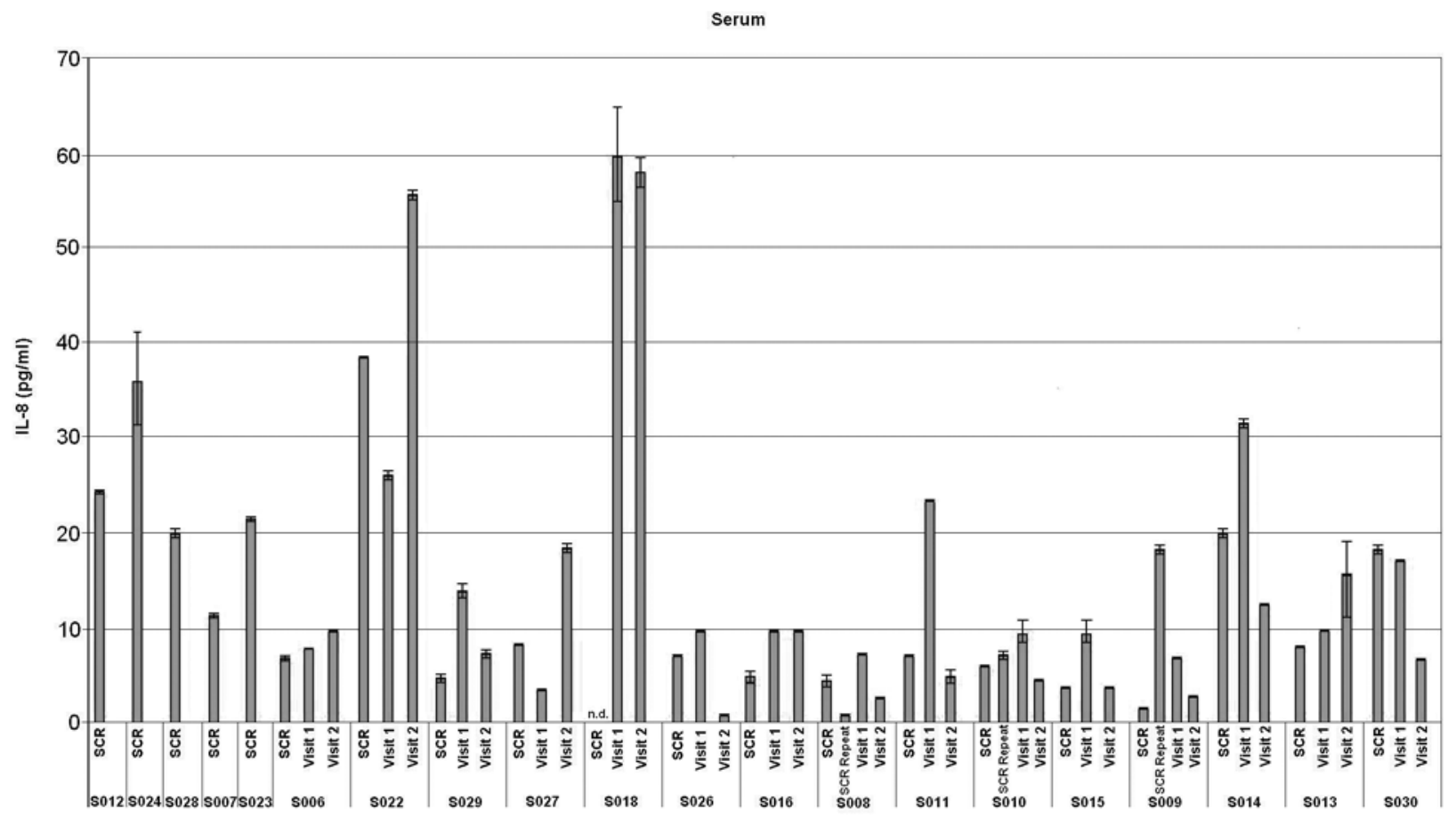

Figure 2. IL-6 serum values. The left part of the figure shows screening data from five patients who could not be enrolled. Then come the IL-6 values of the 15 treated patients, in most cases 3 bars, representing screening, visit 1 and visit 2 . In a few patients, samples had to be repeated, thus they have 4 bars.

Table IV. Immune response at visit 2 and clinical benefit response after 4 weeks.

\begin{tabular}{lll}
\hline $\begin{array}{l}\text { Patients } \\
\text { no. }\end{array}$ & $\begin{array}{c}\text { Immune response } \\
\text { at visit 2 }\end{array}$ & $\begin{array}{c}\text { CBR after 4 weeks } \\
\text { of treatment }\end{array}$ \\
\hline 101 & Decrease & $\mathrm{R}$ \\
102 & No clear effect & $\mathrm{R}$ \\
103 & Increase & $\mathrm{R}$ \\
104 & No clear effect & $\mathrm{ST}$ \\
105 & No clear effect & $\mathrm{R}$ \\
106 & Increase & $\mathrm{NR}$ \\
107 & No clear effect & $\mathrm{NR}$ \\
108 & No clear effect & $\mathrm{NR}$ \\
109 & Decrease & $\mathrm{R}$ \\
110 & Increase & $\mathrm{R}$ \\
111 & Decrease & $\mathrm{NR}$ \\
112 & Increase & $\mathrm{NR}$ \\
113 & Increase & $\mathrm{NR}$ \\
114 & No clear effect & $\mathrm{NR}$ \\
\hline
\end{tabular}

Objective tumor response results. One patient (number 113) had a partial tumor response at week 12 , with a $-40 \%$ change from baseline. Three patients had progressive disease (patients 101 and 110 developed new lesions and patient 114 had a $30 \%$ increase in the sum of longest diameter of the target lesions), and the remaining 11 patients had stable disease (Table II, Fig. 1).
SCC. SCC ranged between 0.5 and over $200 \mu \mathrm{g} / 1$ at both screening and 12 weeks (Table III). Two patients had higher values compared with the other patients (patient 104 had values of $\sim 60 \mu \mathrm{g} / \mathrm{l}$ and patient 105 had values of between $\sim 230$ and $250 \mu \mathrm{g} / \mathrm{l}$ ); both patients had stable disease. One patient (101) had an increase from 21 to $62 \mu \mathrm{g} / 1$ and also had progressive disease according to WHO-RECIST, but otherwise there were no marked trends in SCC tumor marker values and there did not appear to be an association between the SCC concentrations and tumor response.

Immune monitoring. The IL-6 serum values from the blood samples are shown in Fig. 2. The main statement to be drawn from these assessments is, that there is no AMTrelated systemic IL-6 increase during the treatment, which is an important safety feature, since systemic elevation of IL-6 may be detrimental. These data complement the CRP values, where also no systemic increase was noticed.

In summary, it seems that AMT does not result in a nondiscriminatory and non-specific activation of immune cells, which would manifest itself in increasing systemic levels of cytokines, e.g. of IL-6. The immuno-modulatory effects of AMT seem to be locally restricted.

On a cellular level, the cytokine release by isolated white blood cells was, however, affected by AMT. A number of different cytokines were assessed and showed changes throughout the treatment. The full data set will be reported in the integrated study report at the end of the trial. For the present interim report, it is sufficient to say that five patients exhibited an increase of their immune parameters overtime, three a decrease and in the other seven patients there were either no significant changes, or they were inconclusive (e.g. an initial increase followed by a decrease). 
Table V. Tumor response and CBR.

\begin{tabular}{lccc}
\hline Tumor response & No. of patients $(\mathrm{n}=15)$ & $\begin{array}{c}\text { No. of patients who were } \\
\text { clinical responders }\end{array}$ & $\begin{array}{c}\text { No. of patients who were } \\
\text { clinical non-responders }\end{array}$ \\
\hline Partial response & 1 & 1 & 0 \\
Stable & 11 & 7 & 4 \\
Progressive disease & 3 & 0 & 3 \\
\hline
\end{tabular}

Table VI. EORTC quality of life scores.

\begin{tabular}{|c|c|c|c|c|c|c|c|}
\hline \multirow[b]{2}{*}{$(n=15)$} & \multicolumn{7}{|c|}{ Mean score \pm SD } \\
\hline & Baseline & Week 1 & Week 2 & Week 3 & Week 4 & Week 8 & Week 12 \\
\hline $\begin{array}{l}\text { Questions 1-28 } \\
\text { (High score = poor quality of life) }\end{array}$ & $49 \pm 8.3$ & $47 \pm 6.7$ & $40 \pm 5.2$ & $37 \pm 5.6$ & $37 \pm 6.8$ & $37 \pm 7.0$ & $37 \pm 10.7$ \\
\hline $\begin{array}{l}\text { Questions } 29 \text { and } 30 \\
\text { (High score }=\text { good quality of life) }\end{array}$ & $8.8 \pm 3.5$ & $8.8 \pm 2.7$ & $9.3 \pm 1.8$ & $9.9 \pm 2.3$ & $10.1 \pm 2.1$ & $9.8 \pm 1.7$ & $9.9 \pm 2.4$ \\
\hline
\end{tabular}

$C B R$ and tumor response. The patient who had the partial response (number 113), was also a clinical responder, and the three patients with progressive disease were all clinical nonresponders. Of those with stable disease, $64 \%$ were clinical responders, and $36 \%$ were clinical non-responders (Tables IV and V).

Quality of life results. Mean quality of life scores indicated an improvement in the patients' status (Table VI). For questions 1-28, the mean score decreased (i.e. improved) up to week 3 and then remained stable at 37. For questions 29 and 30 , the mean score increased (i.e. improved) up to week 4 and then decreased slightly.

\section{Discussion}

Senegal was selected as a study site due to the large number of advanced inoperable cervical cancer patients which cannot be found in more developed countries due to early screening and treatment. Also Senegal has the necessary medical, clinical, regulatory and ethical infrastructure in place to conduct GCP clinical trials

The safety data showed a good systemic tolerability and no relevant tolerability issues were raised. In spite of the intramuscular route of administration, local reactions at the injection site appeared to be an issue for only 1 patient.

One patient (number 113) had a partial tumor response at week 12 , with a $-40 \%$ change from baseline according to RECIST-criteria. Eleven patients had stable disease with 6 patients having some reduction in tumor which did not qualify as a partial response consistent with RECIST-criteria. Three patients had progressive disease (patients 101 and 110 developed new lesions and patient 114 had a $30 \%$ increase in the sum of longest diameter of the target lesions).
The immunomonitoring data indicated, that although AMT did not induce a general systemic inflammatory response, the cytokine release of certain cells was affected in at least some patients. Due to its immuno-modulatory activities AMT may be able to overcome the immunosuppressive activities of tumors and thus allow the immune system to mount an anti-tumor response. Thus, it was of particular interest to assess a potential correlation between the clinical response and the immune response. A correlation was regarded as such if a clinical responder showed an increase of immune parameters or if a clinical non-responder showed a decrease of immune parameters. Table IV shows the results of this analysis. Since the visit 2 took place at 21-42 days after treatment start, in first instance the immune data were compared to the Clinical Benefit Response after 4 weeks.

There was an indication that there is an association between tumor response at 12 weeks and the CBR, as those patients with progressive disease were all clinical non-responders, and the patient with a partial response was a clinical responder. However, this was a very small sample and no statistical tests of association were performed.

The CBR results may indicate that certain patients may be predisposed to respond to AMT, and others not, as those patients with an initial response tended to have a sustained response over the 3 months of the study, and those who did not respond initially remained non-responders. Three patients with advanced cervical cancer are likely to lose weight without treatment, and therefore the body weight stabilization may indicate a positive effect of the study drug.

This pilot study showed that approximately half of the patients with advanced cervical cancer had a clinical benefit response following 3 months of treatment with AMT. Initial analysis of quality of life data also indicated an improvement. It must of course be considered that these results are from a 
small sample size, in an open-label, non-blinded study, and the duration of treatment was relatively short. The study has now therefore entered the second phase in which 100 patients will be randomized to a controlled study comparing AMT and best supportive care with best supportive care alone. Fifty patients have been recruited so far.

For the main study the primary end point has been changed to progression free survival and the study is now open randomized comparing AMT and best supportive care with best supportive care alone. The study duration is not restricted to 3 months but patients are allowed to continue AMT until the tumor is progressing or the patients experience any limiting toxicity.

\section{Acknowledgements}

We would like to thank the project management team at Focus Clinical Drug Development GmbH, Neuss, Germany, the responsible CRO for this study. We would also like to thank the study team in Dakar, Senegal.

\section{References}

1. Institute for Experimental Oncology, Oncotest $\mathrm{GmbH}, \mathrm{Am}$ Flughafen 12-14, 79108, Freiburg, Germany: In vivo evaluation of Auron-Misheil-Medicine in three different murine tumor models. Protocol No. P82G, (8th December, 2003).

2. Bolli S: Die echte Kamille. Phytotherapie 2: 13-17, 2003.

3. Hudson EA, Dinh PA, Kokubun T, Simmonds MJ and Gescher A: Characterization of potentially chemopreventive phenols in extracts of brown rice that inhibit the growth of human breast and colon cancer cells. Cancer Epidemiol Biomarkers Prev 9: 1163-1170, 2000.

4. Chiang LC, Chiang W, Chang MY, Ng LT and Lin CC: Antileukemic activity of selected natural products in Taiwan. Am J Chin Med 31: 37-46, 2000.

5. Ogiwara T, Satoh K, Kadoma Y, et al: Radical scavenging activity and cytotoxicity of ferulic acid. Anticancer Res 22: 2711-2717, 2002.

6. Mori H, Kawabata K, Matsunaga K, et al: Chemopreventive effects of coffee bean and rice constituents on colorectal carcinogenesis. Biofactors 12: 101-105, 2000.

7. Robinson JB, Singh D, Bodurka-Bevers DC, Wharton JT, Gershenson DM and Wolf JK: Hypersensitivity reactions and the utility of oral and intravenous desensitization in patients with gynecologic malignancies. Gynecol Oncol 82: 550-558, 2001 .

8. Cameron LB: Neuropsychotic drugs as adjuncts in the treatment of cancer pain. Oncology 6: 65-72, 1992.

9. Medina MA, Garcia de Veas R, Morata P, Lozano J and Sánchez-Jiménez FM: Chlorpheniramine inhibits the synthesis of ornithine decarboxylase and the proliferation of human breast cancer cell lines. Breast Cancer Res Treat 35: 187-194, 1995.
10. Gómez-Fabre PM, de Pedro E, Medina MA, Nunez de Castro I and Márquez J: Polyamine contents of human breast cancer cell treated with the cytotoxic agents chlorpheniramine and dihydrodidemnin B. Cancer Lett 113: 141-144, 1997.

11. Wargovich MJ, Jiminez A, McKee K, et al: Efficacy of potential chemopreventive agents on rat colon aberrant crypt formation and progression. Carcinogenesis 21: 1149-1155, 2000.

12. Suessbrich H, Waldegger S, Lang F and Busch AE: Blockade of HERG channels expressed in Xenopus oocytes by the histamine-receptor antagonists terfenadine and astemizole. FEBS Lett 385: 77-80, 1996.

13. Crociani O, Guasti L, Balzi M, et al: Cell cycle-dependent expression of HERG1 and HERG1B isoforms in tumor cells. J Biol Chem 278: 2947-2955, 2003.

14. Argilés JM and Lopéz-Soriano FJ: Why do cancer cells have such a high glycolytic rate? Med Hypotheses 32: 151-155, 1990.

15. Younes RN and Noguchi Y: Pathophysiology of cancer cachexia. Rev Hosp Clin Fac Med Sao Paulo 55: 181-193, 2000.

16. Ayre SG, Garcia y Bellon DP and Garcia DP J: Insulin, chemotherapy, and the mechanisms of malignancy: the design and the demise of cancer. Med Hypotheses 55: 330-334, 2000.

17. Lasalvia-Prisco E, Cucchi S, Vázquez J, Lasalvia-Galante E, Golomar W and Gordon W: Insulin-induced enhancement of antitumoral response to methotrexate in breast cancer patients. Cancer Chemother Pharmacol 53: 220-224, 2004.

18. Pearlstone DB, Wolf RF, Berman RS, Burt M and Brennan MF: Effect of systemic insulin on protein kinetics in postoperative cancer patients. Ann Surg Oncol 1: 321-332, 1994.

19. Minn H, Nuutila P, Lindholm P, Ruotsalainen U, Bergman J, Teras M and Knuuti MJ: In vivo effects of insulin on tumor and skeletal muscle glucose metabolism in patients with lymphoma. Cancer 73: 1490-1498, 1994.

20. Costelli P, Tessitore L, Batetta B, et al: Alterations of lipid and cholesterol metabolism in cachectic tumor-bearing rats are prevented by insulin. J Nutr 129: 700-706, 1999.

21. Lundholm K, Körner U, Gunnebo L, Sixt-Ammilon P, Fouladiun M, Daneryd P and Bosaeus I: Insulin treatment in cancer cachexia: effects on survival, metabolism and physical functioning. Clin Cancer Res 13: 2699-2706, 2007.

22. Therasse P, Arbuck SG, Eisenhauer EA, et al: New guidelines to evaluate the response to treatment in solid tumors, European Organization for Research and Treatment of Cancer, National Cancer Institute of the United States, National Cancer Institute of Canada. J Natl Cancer Inst 92: 205-216, 2000.

23. Aaronson NK, Ahmedzai S, Bergman B, et al: The European Organization for Research and Treatment of Cancer QLQ-C30: a quality-of-life instrument for use in international clinical trials in oncology. J Natl Cancer Inst 85: 365-376, 1993.

24. Scheele J, Drevs J, Diergarten K and Niazi FR: A Phase I double-blind, randomized, placebo-controlled, ascending dose, parallel group trial for the assessment of the safety, tolerability, pharmacokinetic and pharmacodynamic characteristics of single doses of AMT (Auron Misheil Therapy) in healthy male subjects. Int J Pharmac Therap (In press). 